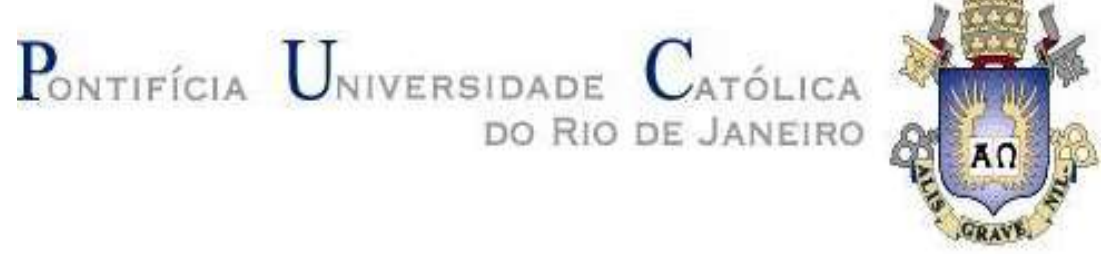

Renzon Daniel Cosme Pecho

Obtenção de substâncias húmicas a partir do rejeito de carvão mineral: Métodos alternativos e caracterização voltada para possíveis aplicações

Tese de Doutorado

Tese apresentada como requisito parcial para obtenção do grau de Doutor pelo Programa de PósGraduação em Engenharia de Materiais e de Processos Químicos e Metalúrgicos do Departamento de Engenharia de Materiais da PUCRio.

Orientador: Prof. Eduardo de Albuquerque Brocchi

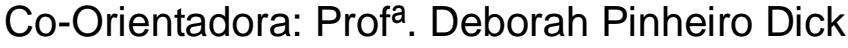


Renzon Daniel Cosme Pecho

\section{Obtenção de substâncias húmicas a partir do rejeito de carvão mineral: Métodos alternativos e caracterização voltada para possíveis aplicações}

Tese apresentada como requisito parcial para obtenção do grau de Mestre pelo Programa de Pósgraduação em Engenharia de Materiais e de Processos Químicos e Metalúrgicos do Departamento de Engenharia de Materiais do Centro Técnico Científico da PUC-Rio. Aprovada pela Comissão Examinadora abaixo assinada.

Prof. Eduardo de Albuquerque Brocchi Orientador e Presidente Departamento de Engenharia de Materiais - PUC Rio

Prof ${ }^{a}$. Deborah Pinheiro Dick Universidade Federal do Rio Grande do Sul- UFRGS

Prof. Raul Almeida Nunez

Pontifícia Universidade Católica do Rio de Janeiro-PUC Rio

Prof. José Brant de Campos Universidade do Estado do Rio de Janeiro-UERJ

Dr. Luís de França da Silva Neto Empresa Brasileira de Pesquisa Agropecuária- Embrapa

Prof. José Eugenio Leal Coordenador Setorial de Pós-Graduação do Centro Técnico Científico da PUC- Rio

Rio de Janeiro, 10 de Dezembro de 2013. 
Todos os direitos reservados. É proibida a reprodução total ou parcial do trabalho sem autorização da universidade, do autor e do orientador.

Renzon Daniel Cosme Pecho

Graduou-se em Engenharia Química pela Universidade Nacional do Centro do Perú em 2005.

Ficha Catalográfica

Cosme Pecho, Renzon Daniel

Obtenção de substâncias húmicas a partir do rejeito de carvão mineral: Métodos alternativos e caracterização voltada para possíveis aplicações / Renzon Daniel Cosme Pecho; orientador Eduardo de Albuquerque Brocchi, co-orientadora Deborah Pinheiro Dick. - 2013.

v. 144 f. : il. (color.); $30 \mathrm{~cm}$

Tese (doutorado)-Pontifícia Universidade Católica do Rio de Janeiro, Departamento de Engenharia de Materiais, 2013.

Inclui bibliografia

1. Engenharia de materiais - Teses. 2. Rejeito de carvão mineral 3. Substâncias húmicas. 4. Ácido húmico. 5. Composição química. 6. Funcionalização. 7. Caraterização. I. Brocchi, Eduardo de Albuquerque. II. Dick, Deborah Pinheiro. III. Pontifícia Universidade Católica do Rio de Janeiro. Departamento de Engenharia de Materiais. IV. Título. 
Dedico meu trabalho a Deus, a minha adorável mãe Alejandrina, a meus irmãos Victor, Jéssica, Tania, Esteban, e meus sobrinhos Lesly, Sarit, Sumi, Ricardo e Sebastian, pelo amor e apoio incondicional nos momentos de felicidade e dificuldade. 


\section{Agradecimentos}

Agradeço a Deus por toda a força nos momentos difíceis, pelas conquistas e pela saúde.

Especial agradecimento ao meu orientador, Professor Eduardo Albuquerque Brocchi, por me acolher desde o primeiro momento que o conheci e confiar nas minhas capacidades para desenvolver o projeto. Agradeço a ele, também, por toda a orientação, pela paciência, carinho, incentivo, pelo valioso apoio acadêmico e moral para a realização deste trabalho. Obrigado por me incentivar, pela valiosa ajuda que me brindou no transcurso do Doutorado.

Agradeço á minha co-orientadora Deborah Dick; muito obrigado pelo grande apoio, por me transmitir seus conhecimentos, pela ajuda valiosa que me brindou no transcurso do doutorado, por confiar em minhas capacidades e por me ensinar a atuar, pensar e trabalhar em equipe, estando sempre presente, corrigindo meus erros em todo momento durante o desenvolvimento deste projeto e por contribuir em muito no meu crescimento acadêmico.

Ao $\mathrm{CNPq}$ e á PUC-Rio, pelo apoio concedido para a realização deste trabalho.

Á professora Rosane Gil do Instituto de Química da UFRJ, por me ensinar o uso de equipamento de espectroscopia de Ressonância Magnética Nuclear e pelo acolhimento em seu laboratório durante as análises de RMN.

À Dr ${ }^{\mathrm{a}}$. Cristiane Portella (LACQ-PUC-RIO), Pelas análises elementares de HCNS.

Ao Técnico Jorge Almeida do departamento de química da PUC-Rio pelas análises de FTIR e DTG. 
Ao Dr. Rogeiro Navarro do DEMa PUC-Rio; pela paciência nas análises de MEV-EDS.

Ao técnico Mauricio do departamento de Química da PUC-Rio pelas análises de ICP-OES.

À Meryelem, pela paciência, carinho, amizade e por toda sua ajuda no doutorado.

Aos Professores, funcionários e colegas do departamento de Engenharia de Materiais da PUC-Rio.

Ao grupo de trabalho de pesquisa pelo suporte nas horas que precisei e por oferecer também seus conhecimentos.

A minha mãe por seu amor e compreensão e porque sempre esteve, quando eu precisava dela.

Aos meus amigos da casa XXI pela amizade e companheirismo. 


\section{Resumo}

Renzon Cosme Pecho; Eduardo de Alburequerque Brocchi (Orientador): Deborah Pinheiro Dick (Co-orientadora). Obtenção de substâncias húmicas a partir do rejeito de carvão mineral: Métodos alternativos e caraterização voltada para possíveis aplicações. Rio de Janeiro, 2013. 144p. tese de Doutorado-Departamento de Engenharia de Materiais, Pontifícia Universidade Católica do Rio de Janeiro.

Este trabalho tem como objetivo principal utilizar um rejeito do processo de concentração de carvão mineral (RJ) como fonte de substâncias húmicas, em particular, do ácido húmico (AH). Para atingir o objetivo a pesquisa foi dividida em três etapas: a obtenção, a caracterização e a aplicação do AH. Na primeira etapa, foram utilizados três métodos diferentes de funcionalização da amostra: o processo químico (PQ), a eletrólise (ELE) e o uso de autoclave (PRESS), previamente ao procedimento de extração das substâncias húmicas $(\mathrm{SH})$, em particular, do ácido húmico (AH). Observou-se que, em termos de recuperação em massa nas condições operacionais estabelecidas, o método de processo químico (PQ) foi o mais eficiente resultando 20,2\% de AH e 3,8\% para AF-Like. Na segunda etapa, estudou-se a caracterização química (acidez fenólica, acidez total, acidez pelos grupos carboxílicos, pH, CTC e cinza), espectroscópica (FTIR, RMN de 13C e ICP-OES), análise elementar, análise térmica (TGA) e análise estrutural (MEV - EDS) dos ácidos húmicos produzidos. Observou-se certa semelhança entre os AH's extraídos do solo e o AH obtido neste trabalho, com a diferença deste ser muito aromático, o grau de aromaticidade mostrou-se muito elevado (em torno de $60 \%$ em relação a $15 \%$ do solo). Além disso, as características em relação ao CTC foram relevantes, pois variou de 22 a $44 \mathrm{Cmolckg}^{-1}$. Este valor é considerado moderadamente alto em relação ao valor médio de $25 \mathrm{Cmolckg}^{-1}$ do CTC, oriundo dos solos brasileiros. Os resultados obtidos por espectroscopia de FTIR e de RMN de 13C indicaram que a funcionalização do rejeito de carvão mineral (RJ) por qualquer um dos métodos de obtenção é relativamente baixa (de 5,0 a 9,7\% C-carboxílico) em comparação aos AH proveniente dos solos. Os resultados comparativos apresentaram similaridade nas características estruturais e físicas, observadas pela microscopia eletrônica de varredura. Na terceira etapa foi realizado um estudo preliminar sobre a capacidade do $\mathrm{AH}$, obtido pelo método $\mathrm{PQ}$ de reter o chumbo, contido em solução sintética $\left(1000 \mathrm{mgL}^{-1}\right)$. Constatando-se 
uma eficiência de $98 \%$ na adsorção deste elemento quando se utilizou uma razão de 1:1(1g AH:1g Pb) e vazão de $0,06 \mathrm{~mL} / \mathrm{min}$.

\section{Palavras Chave}

Rejeito de carvão mineral; Substância Húmica; Ácido Húmico; Composição química; Caracterização. 


\section{Abstract}

Renzon Cosme Pecho; Eduardo Albuquerque Brocchi (Advisor); Deborah Pinheiro Dick (Co-advisor). Extraction of humic substances from tailings of mineral coal beneficiation: Alternative methods and characterization for possible applications. Rio de Janeiro, 2013. 144p. $\mathrm{PhD}$ Thesis - Departamento de Engenharia de Materiais, Pontifícia Universidade Católica do Rio de Janeiro.

This work aims to evaluate the use of a concentration coal tailing (RJ) as a source of humic substances, particularly humic acid (HA). To achieve the goal the research was divided into three steps: obtaining, characterization and application of HA.In the first step three different methods of functionalization of the sample were tested prior to the HA extraction procedure: chemical process (PQ), electrolysis (ELE) and the using of an autoclave (PRESS). It was observed that in terms of mass recovery and within established operating conditions, the method of chemical process (PQ) result was the most efficient, with $20.2 \%$ and $3.8 \%$ of $\mathrm{AH}$ to AF-Like, respectively.In the second step, it was studied the physical and chemical properties of the humic acids produced: phenolic acidity, total acidity, the acidity of carboxyl groups, $\mathrm{pH}, \mathrm{CTC}$ and gray, spectroscopy (FTIR, ${ }^{13} \mathrm{C}$ NMR and ICP - OES), elemental analysis, thermal analysis (TGA) and structural analysis (SEM - EDS). There were some similarities with the AH's extracted from the soil with the difference being the degree of aromaticity which is very high (around $60 \%$ compared to $15 \%$ of the soil). Moreover, the relevant features were the CTC, which ranged $22-44 \mathrm{Cmolckg}^{-1}$. This value is considered moderately high in relation to the approximate mean value of $25 \mathrm{Cmolckg}^{-1} \mathrm{CTC}$, originated from Brazilian soil. The results obtained from FTIR spectroscopy and ${ }^{13} \mathrm{C}$ NMR indicated that functionalization of the coal tailing (RJ), by any of the methods is relatively low (5.0 - 9.7\% C-carboxylic acid) compared to $\mathrm{AH}$ from soil. Also showed similar structural and physical characteristics, as observed by scanning electron microscopy. In the third stage, a preliminary study on the retention capacity of the lead contained in synthetic (1000 ppm) solution by the $\mathrm{AH}$ obtained by the chemical processes method was performed. It was found an 
efficiency of $98 \%$ adsorption of the element when using a ratio of $1: 1(\mathrm{AH} 1 \mathrm{~g}: 1 \mathrm{~g}$ $\mathrm{Pb}$ ) and flow rate of $0.06 \mathrm{ml} / \mathrm{min}$.

\section{Keywords}

Reject coal; humic substance; humic Acid; chemical composition; characteristics. 


\section{Sumário}

1. Introdução 23

2. Objetivos 26

3. Revisão Bibliográfica 27

3.1. Carvão Mineral 27

3.2. Funcionalização da matéria-prima carbonosa 29

3.3. Extração e purificação de substancias húmicas 34

3.4. Substancias húmicas 36

3.5. Caracterizações química e estructural do ácido húmico 39

3.5.1. Infravermelho (FTIR) 39

3.5.2. Análise Termogravimétrico (TG) 39

3.5.3. Ressonância Magnética Nuclear (RMN) 40

3.5.4. Microscopia eletrônica de varredura (MEV-EDS) 41

3.5.5. Análise elementar CHN 42

4. Materiais e Métodos 43

4.1. Etapa I: Funcionalização do rejeito de carvão mineral (RJ) 45

4.1.1. Funcionalização por processo químico (PQ) 46

4.1.2. Funcionalização por eletrooxidação 49

4.1.3. Funcionalização utilizando autoclave 53

4.2. Etapa II: Extração y fracionamento das substâncias húmicas: Obtenção de ácido húmico $(\mathrm{AH})$ e ácido fúlvico like (AF-like) 55

4.3. Etapa III: Caraterização química e estrutural dos ácidos húmicos (AH) obtidos a partir do rejeito de carvão mineral (RJ)

4.3.1. Determinação do teor da acidez total 59

4.3.2. Determinação do teor da acidez carboxilica 60

4.3.3. Determinação do teor da acidez fenólica 61

4.3.4. Determnação do $\mathrm{pH} \quad 61$

4.3.5. Determinação do teor de cinzas 62

4.3.6. Análise de CTC (capacidade de troca catiônica) efetiva 62

4.3.7. Análise elementar 62

4.3.8. Análise por Espectroscopia de FTIR (infravermelho de transmissão com transformada de Fourier) 63

4.3.9. Análise por Termogravimetria (TGA) 65

4.3.10. Espectroscopia de ressonância magnética nuclear do $13 \mathrm{C}$ no estado sólido com polarização cruzada e ângulo mágico com amplitude variável (VACP/MAS RMN 13C)

4.3.11. Microscopia eletrônica de varredura (MEV) acoplada a Espectroscopia por dispersão de energia (EDS)

4.3.12. Determinação de metais por espectrometria de emissão atômica por plasma acoplada indutivamente (OES-ICP)

4.4. Etapa IV: Teste exploratorio para a aplicação do AH na remoção de chumbo $(\mathrm{Pb})$ de efluente sintético 
5.1. Etapas I e II: Eficiência na extração de substancias húmicas (AH e AF-Like) para as diferentes formas de funcionalização 72

5.1.1. Processos químicos

5.1.1.1. PQ: Funcionalização pelo $\mathrm{HNO}_{3}$

5.1.1.2. PQ: Funcionalização com $\mathrm{H}_{2} \mathrm{SO}_{4} \quad 72$

5.1.1.3. PQ: Funcionalização com HCl

5.1.1.4. PQ:Funcionalização com NaOH 74

5.1.1.5. Uma breve comparação entre as funcionalizações por
processos químicos (PQ)

5.1.2. ELE: Funcionalização por eletrooxidação 76

5.1.2.1. ELE/Eletrolito: $\mathrm{H}_{2} \mathrm{SO}_{4}$ efeito da concentração dos ácidos, temperatura e tempo 76

5.1.2.2. ELE/Eletrolito: $\mathrm{H}_{2} \mathrm{SO}_{4}$ efeito da tensão da célula eletrolítica 79

5.1.2.3. ELE/Eletrolito: $\mathrm{H}_{2} \mathrm{SO}_{4}$ efeito do tipo de eletrodo 83

5.1.2.4. ELE/Eletrolito: $\mathrm{NaOH}$ efeito da concentração de $\mathrm{NaOH}$, temperatura e tempo $\quad 84$

5.1.2.5. ELE/Eletrolito: $\mathrm{NaOH}$, efeito da tensão da célula eletrolítica 87

5.1.2.6. Uma breve comparação entre as funcionalizações por Eletrooxidação $\left(\mathrm{H}_{2} \mathrm{SO}_{4}, \mathrm{H}_{2} \mathrm{SO}_{4}+\mathrm{Fe}^{+2}, \mathrm{H}_{2} \mathrm{SO}_{4}+\mathrm{Fe}^{+3}\right.$ e $\left.\mathrm{NaOH}\right) \quad 87$

5.1.3. PRESS: Funcionalização utilizando autoclave 89

5.1.3.1. PRESS/reagente: $\mathrm{NaOH}$. Efeito da concentração e tempo 89

5.1.3.2. PRESS/reagente: $\mathrm{NaOH}$. Efeito da temperatura e pressão 90

5.1.3.3. PRESS/reagente: HCl. Efeito da concentração e tempo 92

5.1.3.4. PRESS/reagente: HCl. Efeito da temperatura e pressão 93

5.1.3.5. PRESS/reagente: $\mathrm{HNO}_{3}$. Efeito da concentração 94

5.1.3.6. Uma breve comparação entre as funcionalizações utilizando autoclave

5.1.4. Comparação das soluções utilizadas entre os diferentes métodos estudados

5.1.5. Comparação da eficiência máxima da cada método (PQ, PRESS e ELE) com aquela obtida pelo método IHSS (para um solo Swift, (1996))

\subsection{Caracterização química e estructural dos materiais envolvidos: rejeito de carvão mineral e ácido húmico extraído}

5.2.1. Rejeito de carvão mineral (RJ)

5.2.1.1. Composição básica e análise elementar 98

5.2.1.2. Otras características físico-químicas do RJ 100

5.2.2. Ácido Húmico extraido 100

5.2.2.1. Composição química e acidez 100

5.2.2.2. Espectros de Infravermelho (FTIR) 105

5.2.2.3. Índices e intensidades relativas obtidos dos espectros de FTIR 108

5.2.2.4. Análise por espectroscopia de ressonância magnética nuclear ${ }^{13} \mathrm{C}(\mathrm{CP} / \mathrm{MAS} \mathrm{RMN}) \quad 109$

5.2.3. Comparação entre o AH extraído e o RJ 113 
5.2.3.1. Descomposição Térmica

5.2.3.2. Caraterização por microscopia de varredura coplada ao EDS (MEV-EDS)

5.3. Etapa III: Teste exploratorio para aplicação do AH extraido no tratamento de efluentes para remoção de chumbo

5.3.1. Adsorção de chumbo $(\mathrm{Pb})$ no $\mathrm{AH}$

$\begin{array}{lll}\text { 6. Conclusões } & 124\end{array}$

7. Recomendações para trabalhos futuros 127

$\begin{array}{lll}\text { 8. } & \text { Referências } & 128\end{array}$

9. Apêndice 140

9.1. Apêndice 1 140

9.2. Apêndice 2 141

9.3. Apêndice 3 142

9.4. Apêndice 4 142

9.5. Apêndice 5 143 


\section{Lista de Figuras}

Figura 1 Tipos de carvões minerais e principais usos. (World Coal Institute por DNPM/DIDEM, 2008) 28

Figura 2 Mecanismo de oxidação por procesos quimicos (PQ). 31

Figura 3 Modelo hipotético de uma macromolécula de carvão betuminoso, (CARLSON et al., 1992).

Figura 4 Fracionamento de substâncias húmicas com base em características e solubilidade (STEVENSON, 1994).

Figura 5 Ilustração do procedimento experimental da extração de $\mathrm{AH}$ e AF-Like e métodos de caracterização.

Figura 6 Conjunto de placa aquecedora, erlenmeyer com Tubo de refluxo acoplado em serie.

Figura 7 Esquema simplificado para obtenção de AH e AF-Like. Funcionalização via o processo químico (PQ).

Figura 8 Célula eletroquímica com os compartimentos para o cátodo, ânodo e eletrodo de referência (SCE), acoplado a condensadores.

Figura 9 Esquema simplificado da produção de $\mathrm{AH}$ e AF-Like. Funcionalização via ELE.

Figura 10 Reator PARR 4842, com compartimentos de pressão e o controle automatizado de temperatura e pressão.

Figura 11 Esquema simplificado da produção de AH e AF-Like. Funcionalização via alta pressão (PRESS)

Figura 12 Equipamento de titulação para análise do teor da acidez total e acidez carboxílica. (1; agitador, 2; bureta, 3; medidor de $\mathrm{pH}, 4$; balão de fundo chato com 3 aberturas).

Figura 13 Exemplo do espectro FTIR com intensidades relativas calculadas.

Figura 14 Esquema dos experimentos de adsorção em fluxo continuo. 
Figura 15 Eficiência na extração de $\mathrm{AH}$ e AF-Like em função do tempo para diferentes concentrações de $\mathrm{HNO}_{3}$ e razão solido (RJ) /solução (tamanho de partícula; $<0,057 \mathrm{~mm}$ )

Figura 16 Eficiência da extração de $\mathrm{AH}$ e $\mathrm{AF}$ em função do tamanho de partícula $\left(\mathrm{HNO}_{3} 40 \%\right.$, 5h de reação e sólido/solução 1:20).

Figura 17 Eficiência na extração de $\mathrm{AH}$ e AF-Like em função da concentração de $\mathrm{H}_{2} \mathrm{SO}_{4}$ (temperatura de borbulhamento, tempo de reação $5 \mathrm{~h}$, solida/solução 1:20 e tamanho de partícula <0,057 mm).

Figura 18 Eficiência na extração de $\mathrm{AH}$ e AF-Like em função da concentração de $\mathrm{HCl}$ (temperatura de borbulhamento, tempo de reação $5 \mathrm{~h}$, solida/solução 1:20 e tamanho de partícula <0,057 mm).

Figura 19 Eficiência na extração de AH e AF-Like em função da concentração de $\mathrm{NaOH}$ (temperatura de borbulhamento, tempo de reação $5 \mathrm{~h}$, solida/solução 1:20 e tamanho de partícula <0,057 mm).

Figura 20 Relação entre a eficiência na produção de AH e AF-Like e as diferentes soluções empregadas no método PQ(temperatura de borbulhamento, tempo de reação $5 \mathrm{~h}$, solida/solução 1:20 e tamanho de partícula $<0,057 \mathrm{~mm})$.

Figura 21 Eficiência na produção de AH pela eletrooxidação do RJ em função do tempo de reação para diferentes concentrações de $\mathrm{H}_{2} \mathrm{~S}_{4}$ e temperaturas (1,2 V vs SCE e tamanho de partícula de 0,057).

Figura 22 Eficiencia na produção de $\mathrm{AH}$ e AF-Like em função da tensão da célula (temperatura de $80^{\circ} \mathrm{C}$, com solução de $\mathrm{H}_{2} \mathrm{SO}_{4} 40 \%$, sólido/solução $0,015 \mathrm{~g} \mathrm{RJ} / \mathrm{mL}$, tamanho das particulas de $<0.057 \mathrm{~mm}$, tempo de reação de $12 \mathrm{~h}$ e eletrodo de Pt.

Figura 23 Relação da densidade de corrente com as soluções de $\mathrm{H}_{2} \mathrm{SO}_{4}$ de $40 \%$ com adição de $\mathrm{Fe}^{+3}$ e $\mathrm{Fe}^{+2}$, nas tensões de $0.8,1,1.2 \mathrm{~V}$ (eletrodo de Pt, tamanho de partícula $<0,057 \mathrm{~mm}$ ).

Figura 24 Representação esquemática do mecanismo da eletrooxidação do carvão com adição de $\mathrm{Fe}^{+3}$ e $\mathrm{Fe}^{+2}$

Figura 25 Relação da tensão (a) e da corrente (b) com o tempo de polarização nas soluções de $\mathrm{H}_{2} \mathrm{SO}_{4} 40 \%$ e $\mathrm{H}_{2} \mathrm{SO}_{4} 40 \%+\mathrm{Fe}^{+3}, \mathrm{Fe}^{+2}$ de $4 \cdot 10^{-6} \mathrm{M}, \mathrm{T}=80^{\circ} \mathrm{C}$, sólido/solução de $0,02 \mathrm{~g} \mathrm{RJ} / \mathrm{mL}$. 
Figura 26 Desempenho da eletrooxidação do RJ nos eletrodos Pt e $\mathrm{Pt} / \mathrm{Ir}$ a) na eficiência de $\mathrm{AH}$ e b) densidade de corrente, com a solução de $\mathrm{H}_{2} \mathrm{SO}_{4} 40 \%+\mathrm{Fe}^{+2} 4.10^{-6} \mathrm{M}$, tensão $1,2 \mathrm{~V}$, tamanho de partícula $<0,057 \mathrm{~mm}$ e temperatura de $80^{\circ} \mathrm{C}$.

Figura 27 Eficiência da produção de $\mathrm{AH}$ em função da concentração de $\mathrm{NaOH}$, (temperatura de $80^{\circ} \mathrm{C}$, sólido/solução de $0,02 \mathrm{~g} \mathrm{RJ} / \mathrm{ml}$, tensão $1,2 \mathrm{~V}$, tempo de reação $12 \mathrm{~h}$ e tamanho de partícula $<0,057 \mathrm{~mm}$ ).

Figura 28 Eficiência na produção de $\mathrm{AH}$ em função da temperatura de reação, (tensão de 1,2V, sólido/solução de 0,02 g RJ/ml, tamanho de partícula $<0,057 \mathrm{~mm}, 12 \mathrm{~h}$ tempo de reação e concentração de $\mathrm{NaOH} 1 \mathrm{M})$.

Figura 29 Eficiência na produção de $\mathrm{AH}$ em função do tempo de reação (temperatura de $80^{\circ} \mathrm{C}$, sólido/solução de $0,02 \mathrm{~g} \mathrm{RJ} / \mathrm{ml}$, tensão de $1,2 \mathrm{~V}$, tamanho de partícula $<0,057 \mathrm{~mm}$ e solução de $\mathrm{NaOH} 1 \mathrm{M})$.

Figura 30 Eficiência na produção de $\mathrm{AH}$ em função da tensão na célula $\left(80^{\circ} \mathrm{C}\right.$, sólido/solução de $1 / 50 \mathrm{~g} \mathrm{RJ} / \mathrm{mL}$, tamanho de partícula $<0.057$ mm, tempo de reação 12 h e solução de $\mathrm{NaOH} 1 \mathrm{M}$ ).

Figura 31 Eficiência na produção de $\mathrm{AH}$ em função dos métodos eletroquímicos nas diferentes soluções testadas (temperatura de $80^{\circ} \mathrm{C}$, sólido/solução de 0,02 $\mathrm{g} \mathrm{RJ} / \mathrm{ml}$, tamanho de partícula <0,057 mm e tempo de reação de $12 \mathrm{~h}$ ).

Figura 32 Eficiência na produção de $\mathrm{AH}$ em função do tempo de reação (concentrações de $\mathrm{NaOH}$, (temperatura de $200^{\circ} \mathrm{C}$, pressão 18 bar, sólido/solução 1:20 e tamanho de partícula <0,057 mm).

Figura 33 Eficiência na produção de $\mathrm{AH}$ em função de a) temperatura e b) pressão (tempo de reação de $5 \mathrm{~h}$, tamanho de partícula $<0,057 \mathrm{~mm}$ e na solução de $\mathrm{NaOH}$ de $0,5 \mathrm{M})$.

Figura 34 Eficiência na produção de $\mathrm{AH}$ e AF-Like em função da concentração da $\mathrm{HCl}$ (temperatura de $200^{\circ} \mathrm{C}$, tempo de reação de $5 \mathrm{~h}$, tamanho de partículas < 0,057 mm e pressão 18 bar).

Figura 35 Eficiência na produção de $\mathrm{AH}$ e AF-Like em função ao tempo de reação, na solução de $\mathrm{HCl}$ de 0,5 M (tamanho de partículas $<0,057 \mathrm{~mm}$, temperatura de $200^{\circ} \mathrm{C}$ e pressão 18 bar) 
Figura 36 Eficiência da produção de AH e AF-Like em função da: a) temperatura, b) pressão ( $\mathrm{HCl}$ 0,5M,tamanho de partícula $<0,057 \mathrm{~mm}$ e tempo de reação de $5 \mathrm{~h})$.

Figura 37 Eficiência na produção de $\mathrm{AH}$ e AF-Like entre as diferentes soluções no método pressão (PRESS) (temperatura de 200 ${ }^{\circ} \mathrm{C}$, pressão de 18 bar, tempo de reação de 5 h e tamanho de partícula $<0,057 \mathrm{~mm})$.

Figura 38 Efeito da solução utilizada na extração de AH e AF-Like para todos os métodos estudados (PQ, PRESS e ELE).

Figura 39 Comparação da eficiência máxima de extração do $\mathrm{AH}$ e AF-Like em função dos métodos PRESS, PQ e ELE e IHSS (SWIFT, 1996).

Figura 40 Correlação da razão atômica de H:C com os grupos carboxílicos dos AH produzidos pelos métodos (PQ, ELE e PRESS).

Figura 41 Correlação do teor de N\% com razão H:C.

Figura 42 Avaliação dos diferentes tipos de materiais no diagrama de Van Krevelen dos AH produzidos pelos métodos ELE, PRESS e PQ.

Figura 43 Espectros de FTIR dos AH obtidos pelos métodos PQ, ELE e PRESS em relação ao RJ.

Figura 44 Correlação das funções oxidadas (C-fenólico + C-carboxil + C-carbonil) com o CTC dos AH produzidos pelos métodos PQ, ELE e PRESS.

Figura 45 Espectros de CP/MAS 13C NMR dos HA obtidos pelos métodos PQ, ELE e PRESS a partir do RJ

Figura 46 Termogramas dos Ah obtidos pelos métodos PQ, ELE e PRESS e do RJ.

Figura 47 Micrografias obtidas no $\mathrm{MEV}$ (A) do RJ; (B) do $\mathrm{AH}$ (NaOH-PQ); (C) do $\mathrm{AH}\left(\mathrm{A}-\mathrm{HNO}_{3}-\mathrm{PQ}\right)$; (D) do $\mathrm{AH}\left(\mathrm{B}-\mathrm{HNO}_{3}-\mathrm{PQ}\right)$; (E) do $\mathrm{AH}(\mathrm{HCl}-\mathrm{PQ})$; (F) do $\mathrm{AH}\left(\mathrm{H}_{2} \mathrm{SO}_{4}-\mathrm{PQ}\right)$, precipitado em $\mathrm{pH}<2$

Figura 48 Micrografias obtidas no $\mathrm{MEV},(\mathrm{G})$ do $\mathrm{AH}\left(\mathrm{H}_{2} \mathrm{SO}_{4}\right.$-ELE); (H) $\mathrm{AH} \quad\left(\mathrm{ELE}_{2} \mathrm{H}_{2} \mathrm{SO}_{4}-\mathrm{Fe}^{+2}\right) ;$ (I) $\mathrm{AH}\left(\mathrm{ELE}_{2} \mathrm{H}_{2} \mathrm{SO}_{4}-\mathrm{Fe}^{+3}\right) ;$ (J) $\mathrm{AH}$ (NaOH-ELE) e (K) precipitado no cátodo com sua EDS. 
Figura 49 Micrografias obtidas no MEV, (L) AH (HCl-PRESS) e (M) $\mathrm{AH}(\mathrm{NaOH}-\mathrm{PRESS})$.

Figura 50 Relação de remoção de $\mathrm{Pb}^{+2}$ e as proporções molares de $\mathrm{AH}$, numa concentração inicial de $\mathrm{Pb}^{+2} 1000 \mathrm{ppm}$, no $\mathrm{pH}<2$, vazão de $0,06 \mathrm{~mL} / \mathrm{min}$ e $0,67 \mathrm{~mL} / \mathrm{min}$.

Figura A5 Espectros de SEM-EDS dos AH e RJ. 


\section{Lista de Tabelas}

Tabela 1 Balanço de massa do carvao na produção de $\mathrm{AH}$ com $\mathrm{NaOH}$ como eletrolito na electrooxidação.

Tabela 2 Composição Elemental dos Ácidos Húmicos (AH) e ácidos

Fúlvicos (AF) extraídos do solo. (teor media).

Tabela 3 Níveis das variáveis independentes do teste de otimização do processo de produção de substância húmica com solução de $\mathrm{HNO}_{3}$.

Tabela 4 Níveis das variáveis independentes do teste de funcionalização por eletrolises em $\mathrm{H}_{2} \mathrm{SO}_{4}$.

Tabela 5 Níveis das variáveis independentes do teste de funcionalização utilizando autoclave com $\mathrm{NaOH}$.

Tabela 6 Resultados da análise estatística dos parâmetros de repetitividade $(\operatorname{Re}),\left(\mathrm{C}_{\mathrm{v}}\right)$, erro $(\mathrm{E})$ e $\left(\mathrm{U}_{\mathrm{x}}\right)$, do método PQ com diferentes soluções no pré-tratamento.

Tabela 7 Resultados da análise estatística: parâmetros de repetitividade $(\mathrm{Re}), \mathrm{Cv}$, erro $(\mathrm{E})$ e incerteza padrão $\left(\mathrm{U}_{\mathrm{x}}\right)$ (método eletroquímico nas diferentes soluções).

Tabela 8 Resultados da análise estadística: repetitividade $(\operatorname{Re})$, coeficiente de variação $(\mathrm{Cv})$, erro e Incerteza $\mathrm{U}_{\mathrm{x}}$ para o método de alta pressão (PRESS).

Tabela 9 Composição básica do RJ

Tabela 10 Análise elementar do RJ por OES-ICP 99

Tabela 11 Algumas características físico-químicas do RJ

Tabela 12 Composição elementar, razões atômicas, cinzas e grupos de ácidos (carboxílico, fenólicos e totais) dos AH extraidos.

Tabela 13 Intensidades relativas e índices de aromaticidade (IA) obtidos dos espectros de FTIR dos AH produzidos pelos métodos PQ, ELE e PRESS

Tabela 14 Distribuição percentual dos tipos de $\mathrm{C}$ dos $\mathrm{AH}$ obtidos pelos métodos (PQ, ELE e PRESS), determinadas nos espectros de RMN, e calculo do grau de aromaticidade. 
Tabela 15 Perda de massa (PP) na análise termogravimétrica em quatro intervalos de temperatura e massa do resíduo para $\mathrm{T}=900^{\circ} \mathrm{C}$ e índice termogravimétrico (ITG) dos $\mathrm{AH}$ obtidos pelos métodos PQ, ELE e PRESS.

Tabela 16 Resultados da composição dos principais elementos encontrados na superfície do RJ e dos AH pelo método EDS-MEV.

Tabela 17 Resultados do EDS-MEV da composição média dos AHPQ, AH-PRESS e AH-ELE e os resultados do RJ final após da oxidação e extração.

Tabela A1 Dados da produção de AH pelo método PQ na solução de $\mathrm{HNO}_{3}$.

Tabela A2 Dados da produção de AH pelo método ELE na solução de $\mathrm{H}_{2} \mathrm{SO}_{4}$.

Tabela A3 Dados da produção de AH pelo método PRESS na solução de $\mathrm{NaOH}$

Tabela A4 Dados da produção de AH pelos métodos PQ, ELE e PRESS nas soluções de $\mathrm{NaOH}, \mathrm{HCl}, \mathrm{H}_{2} \mathrm{SO}_{4}, \mathrm{Fe}^{+2}, \mathrm{Fe}^{+3}$ e $\mathrm{HNO}_{3}$ obtidos com os parâmetros otimizados, para o calculo das variáveis estatísticas (a) AH e (b)AF-Like. 


\section{Lista de siglas e abreviaturas}

\begin{tabular}{|c|c|}
\hline $\mathrm{AH}$ & Ácido Húmico \\
\hline AF- Like & Ácido fúlvico like \\
\hline $\operatorname{Re}$ & Repetitividade \\
\hline $\mathrm{Cv}$ & Coeficiente de variação \\
\hline Ux & Incerteza padrão. \\
\hline PQ & Método Processos Químicos \\
\hline ELE & Método Eletroquímico \\
\hline PRESS & Método de alta Pressão ou em autoclave \\
\hline TG & Termogravimétria \\
\hline ITG & Índice termogravimétrico \\
\hline DTG & Primeira derivada termogravimétrica \\
\hline FTIR & Infravermelho com transformada de Fourier \\
\hline${ }^{13} \mathrm{C} \mathrm{VACP} / \mathrm{N}$ & $\begin{array}{ll}\text { AS RMN } & \text { Ressonância magnética nuclear de carbono } 13 \text { com } \\
& \text { polarização cruzada em torno do ângulo mágico com } \\
& \text { amplitude variável }\end{array}$ \\
\hline MEV-EDS & Microscopia eletrônica de varredura com Scanning Electron Microscopy. \\
\hline ICP-OES & Indução de plasma acoplada com emissão ótica. \\
\hline RJ & Rejeito de carvão mineral \\
\hline SCE & Eletrodo de referência de calomelano \\
\hline h & Hora \\
\hline $\mathrm{V}$ & Tensão \\
\hline Mesh & Unidade de medida do tamanho de partículas \\
\hline IHSS & Sociedade internacional de substancias húmicas \\
\hline IA & Índice de aromaticidade \\
\hline $\mathrm{IH}$ & Índice de hidrofobicidade \\
\hline $\mathrm{S}_{\mathrm{n}}$ & Desvio padrão \\
\hline $\mathrm{n}$ & numero de dados \\
\hline $\mathrm{t}_{95 \%}$ & T- student para um intervalo de confiança do $95 \%$ \\
\hline $\mathrm{E}$ & Erro \\
\hline $\mathrm{x}$ & Média dos dados \\
\hline $\mathrm{t}$ & tempo \\
\hline $\mathrm{T}$ & Temperatura \\
\hline
\end{tabular}


$\mathrm{Pt} / \mathrm{Ir} \quad$ Eletrodo de Platina/Irídio

CTC Capacidade de troca catiônica

$\mathrm{C}_{\mathrm{C}} \quad$ Concentração de carbono

EPA Agencia de proteção ambiental dos Estados Unidos

$\mathrm{R}^{2} \quad$ Coeficiente de determinação

$\mathrm{CC} \quad$ Corrente-continua

CA Corrente-alternada

AH-RJ Ácido húmico produzido a partir de rejeito de carvão mineral

MOS Material orgânico do solo 